are all similar to the whole triangle. Now $P R$ and $Q R$ are equally inclined to $A D$ (a pedal property), and $N$ is the image of $Q$ in $A D$. $\therefore P R$ produced passes through $N$; similarly $L M$ produced passes through $Q$. Let these lines cut $B C$ in $S$ and $S^{\prime}$.

Again $P N \| D B$ since alternate angles $B D R, D R P$ are corresponding angles of the similar triangles $B D A, D R P$; similarly $L Q \| D C$.

$$
\text { But } \begin{aligned}
B S: S C & =D P: P C & & (B D \text { parallel to } S P) \\
& =B L: L D & & \text { (complete similarity of the figures) } \\
& =B S^{\prime}: S^{\prime} C & & \left(L S^{\prime} \text { parallel to } D C\right)
\end{aligned}
$$

$\therefore S$ and $S^{\prime}$ coincide.

Now area of triangle $N B S=$ area of triangle $N D S$ ( $N S$ parallel to $B D$ )

$$
\text { , } \quad Q C S=\quad, \quad, \quad Q D S(Q S \quad, \quad D C) .
$$

To the sum of these areas add area $A N S Q$.

$\therefore$ in area, triangle $A B C=$ kite $A N D Q=$ twice triangle $A N D$.

G. D. C. STokes.

Editor's Notw.-Mr John T. Brown suggests the following neat method of proving that the triangle $A B C$ is twice the triangle $A N D$ :

Suppose $D N$ produced its own length to $E$.

Then the angles $E A D, B A C$ are equal,

$$
\text { and } A E \cdot A D=A J^{2}=A B \cdot A C \text {. }
$$

Hence, by Euc. VI, 15, the triangles $A E D, A B C$ are equal; i.e. twice triangle $A N D=$ triangle $A B C$.

$$
\text { W. A. }
$$

\title{
A Proof of the Theorem of Pythagoras.
}

The triangle $A B C$ has a right angle at $B$. On $A C$, on the same side as $B$, describe a square $A D E C$. Draw $D F$ perpendicular to $A B$ or $A B$ produced.

The triangles $A B C$ and $D F A$ are congruent, having sides $C A$ and $A D$ equal, and the corresponding angles equal. Hence $D F$ is equal to $A B$. 
xiv

Join $D B$ : then the triangle $A D B$ is equal to half the rectangle with $A B$ as base and $F D$ as altitude; that is, to half the square on $A B$.

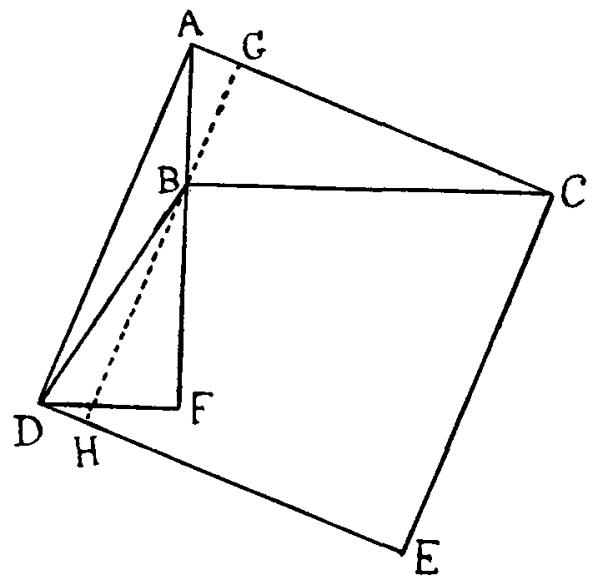

Now through $B$ draw $G B H$ parallel to $A D$ to meet $A C$ in $G$ and $D E$ in $H$. Then the rectangle $A D H G$, being equal to twice the triangle $A D B$, is equal to the square on $A B$.

Similarly the rectangle $C G H E$ is equal to the square on $B C$. Thus, on adding, we find that the square on $A C$ is equal to the sum of the squares on $A B$ and $B C$.

T. M. MacRobert.

\section{Note on a Vanishing Determinant.}

1. In one of Sir Thomas Muir's more recent historical papers on the theory of determinants (Proc. R.S. Edin., XLIII, 1922, p. 129), is included a result due to V. Jung, commented on as being "verified in an unsuggestive way for the first three cases." The theorem is that the determinant

$$
\begin{array}{ccccc}
1 & 1^{2} & \ldots & 1^{n} & 1^{n+1} \\
2 & 2^{2} & \ldots & 2^{n} & 2^{n+1} \\
\ldots & \cdots & \ldots & \cdots & \cdots \\
n & n^{2} & \ldots & n^{n} & n^{n+1} \\
n & \frac{n^{2}}{3} & \ldots & \frac{n^{n}}{n+1} & \frac{n^{n+1}}{n+2} \\
2 & 3 & & n
\end{array}
$$

vanishes when $n$ is even. Below we give a simple proof. 